\title{
OBSESIVIDAD E INTRUSIONES ALIMENTARIAS EN PACIENTES CON TRASTORNOS ALIMENTARIOS Y EN LA POBLACIÒN GENERAL
}

\author{
MARÍA RoNCERO ${ }^{1}$, CONXA PERPIÑÁ ${ }^{1,2}$ y AMPARO BELLOCH ${ }^{1}$ \\ ${ }^{1}$ Facultad de Psicología, Universidad de Valencia \\ ${ }^{2}$ Ciber Fisiopatología Obesidad y Nutrición, Instituto de Salud Carlos III, España
}

\begin{abstract}
Resumen: Los trastornos alimentarios (TA) y el trastorno obsesivo-compulsivo (TOC) son entidades clínicas diferenciadas, aunque comparten elementos psicopatológicos comunes poco investigados. Nuestros objetivos son, primero, analizar en población general $(n=100)$ y personas con TA $(n=84)$ la relación entre síntomas TOC e intrusiones cognitivas de contenido alimentario, su impacto emocional, y las estrategias para controlarlas; segundo, examinar en qué medida los síntomas TOC predicen esas intrusiones. Los resultados muestran, en población general, asociaciones significativas entre síntomas de TOC y frecuencia de intrusiones sobre ejercicio físico, consecuencias emocionales, valoraciones disfuncionales, y estrategias de control, que se mantienen tras controlar edad, índice de masa corporal, depresión, y ansiedad. En pacientes TA, las asociaciones con síntomas TOC únicamente se mantuvieron con consecuencias emocionales y valoraciones disfuncionales. Los resultados indican que las relaciones entre ambos trastornos se explican por la importancia otorgada a las experiencias cognitivas desagradables, y no tanto a la frecuencia con que las experimentan.
\end{abstract}

Palabras clave: Trastornos alimentarios, intrusiones mentales, síntomas obsesivo-compulsivos.

Osessionality and eating intrusions in patients with eating disorders and the general population

\begin{abstract}
Eating disorders (ED) and obsessive-compulsive disorder (OCD) are different clinical entities, although they share psychopathological elements that have been insufficiently investigated. Our objectives are firstly to analyze, in patients with ED, relationship between OCD symptoms and cognitive intrusions with eating content, their emotional impact, and strategies for controlling them and, secondly, to examine to what extent OCD symptoms predict these intrusions in patients with ED. In the general population, results showed significant associations between OCD symptoms and frequency of intrusions on physical exercise, emotional consequences, dysfunctional appraisals, and control strategies, which persisted after controlling for age, body mass index, depression, and anxiety. In ED patients, associations with OCD symptoms persisted only for emotional consequences and dysfunctional appraisals. These results indicate that the relationship between both disorders is explained by the importance that patients give to the unpleasant cognitive experiences, rather than by the frequency with which these are experienced.
\end{abstract}

Keywords: Eating disorders, mental intrusions, obsessive-compulsive symptoms.

\section{INTRODUCCIÓN}

Recibido: 18-mayo-2010; Aceptado: 28-junio-2010

Correspondencia: María Roncero Sanchís Departamento de Personalidad, Evaluación y Tratamientos Psicológicos. Facultad de Psicología. Universidad de Valencia. Av. Blasco Ibáñez, 21. 46010. Valencia (España). E-mail: Maria.roncero@uv.es

Agradecimientos: Investigación financiada en parte por el Ministerio de Educación (Refs.: SEJ2006/03893-PSIC y PSI2009-10957), y en parte por La Conselleria de Empresa, Universidad y Ciencia, Generalitat Valenciana (AE/07/022).
La definición y categorización diagnóstica actual de los trastornos alimentarios (TA) establece una diferenciación nítida entre éstos y el trastorno obsesivo compulsivo (TOC). Así, entre las recomendaciones del DSM-IV-TR (APA, 2000) para el diagnóstico diferencial de la anorexia nerviosa (AN) con el TOC se alude a diferencias de contenido: si el contenido de las obsesiones y compulsiones en la AN refiere a temática alimentaria o similares, el diagnóstico 
debe ser de TA y, en ningún caso, de TOC. Algo similar sucede con uno de los criterios diagnósticos para el TOC en el mencionado manual: se indica que si este trastorno es comórbido con un TA, las obsesiones y/o compulsiones no deben hacer referencia a preocupaciones por la comida, pues en ese caso, habría que establecer un diagnóstico diferencial para delimitar cuál de los dos trastornos, el TA o el TOC, es el principal. Más allá de la utilidad de este tipo de criterios y los debates sobre su adecuación o viabilidad, lo que subyace es, a pesar de lo que pueda parecer, el reconocimiento implícito de que entre ambos conjuntos de trastornos existen más similitudes de las que cabría esperar, especialmente desde un punto de vista psicopatológico.

Por lo que se refiere al TOC, desde la investigación pionera de Rachman y de Silva (1978) y su verificación en múltiples estudios y contextos, hay un acuerdo prácticamente unánime en considerar que el elemento psicopatológico central del trastorno son los pensamientos, imágenes, impulsos, o sensaciones intrusos desagradables y recurrentes sobre un conjunto determinado de temas: sexo, agresión, contaminación, orden, dudas/comprobación, superstición/magia (García-Soriano, Belloch, y Morillo, 2008).

Por su parte, las personas con TA experimentan pensamientos, imágenes y/o impulsos intrusos y repetitivos, cuya temática versa sobre el aspecto físico, el peso, la dieta, y el ejercicio físico. Estas intrusiones cognitivas capturan recursos atencionales conscientes que interfieren con las tareas cotidianas y pueden resultar molestas y difíciles de controlar (Sánchez-Reales, 2009; Serpell, Treasure, Teasdale, y Sullivan, 1999). Además, esta modalidad de intrusiones se observa también en la población general, aunque su frecuencia es menor, al igual que sus consecuencias y/o repercusiones emocionales (Perpiñá, Roncero, y Belloch, 2008), lo que indica que se trata de acontecimientos mentales muy generalizados, y por lo tanto, deberían ser considerados desde una perspectiva dimensional. Las personas con TA ponen en marcha conductas compensatorias funcionalmente relacionadas con el contenido de esas intrusiones (i.e., restringen la dieta, hacen ejercicio físico de manera inapropiada o excesiva, se pesan, etc.) y, en ocasiones, las realizan de manera ritualizada. También hacen uso de otras estrategias, tales como intentar suprimir o mantener bajo control las intrusiones molestas. En definitiva, tanto las denominadas conductas compensatorias, como los intentos por suprimir las intrusiones, poseen una finalidad similar a las estrategias de neutralización manifiestas o encubiertas, ritualizadas o no, que ponen en marcha muchos pacientes con TOC: atenuar el malestar que generan las intrusiones (Sánchez-Reales, 2009; Serpell, Livingstone, Neiderman, y Lask, 2002; Soetens, Braet, Dejonckheere, y Roets, 2006; Woolrich, Cooper, y Turner, 2008). Hay un buen número de investigaciones que evidencian una relación importante entre el TOC y los TA. Por ejemplo, los estudios de comorbilidad en pacientes con TA indican una prevalencia elevada no sólo de TOC de la personalidad (Crane, Roberts, y Treasure, 2007; Thornton y Russell, 1995; Swinbourne y Touyz, 2007), sino también de TOC a nivel de síntomas y de síndromes, excluyendo como indica el DSM-IV-TR, las preocupaciones relacionadas con la imagen corporal, la dieta, o el peso (Halmi et al., 2003; Serpell et al., 2002; Speranza et al., 2001). El subtipo de obsesiones y/o compulsiones más prevalentes tanto en AN como en Bulimia Nerviosa (BN) son las de orden y simetría (Bastiani et al., 1996; Matsunaga et al., 1999a; Matsunaga et al., 1999b). La presencia de síntomas TOC se ha relacionado con el bajo peso y con una mayor gravedad del TA (Davies, Liao, Campbell, y Tchanturia, 2009; Humphreys, Clopton, y Reich, 2007), y no parece que los síntomas obsesivos se puedan atribuir al estado de inanición de las pacientes, puesto que se ha observado que éstos persisten tras la recuperación del TA (Morgan, Wolfe, Metzger, y Jimerson, 2007; Pollice, Kaye, Greeno, y Weltzin, 1997; Von Ranson, Kaye, Weltzin, Rao, y Matsunaga, 1999). Además, se ha planteado que la presencia de síntomas TOC en pacientes con TA podría ser un predictor de las recaídas en el trastorno alimentario (Carter, Blackmore, Sutandar-Pinnock, y Woodside, 2004). Los síntomas TOC se han relacionado también con un mal pronóstico del 
TA (Milos, Spindler, Ruggiero, Klaghofer, y Schnyder, 2002), aunque no en todos los estudios (p.ej., Thiel, Zuger, Jacoby, y Schubler, 1998).

En contraste con la cantidad de investigaciones que muestran la existencia de comorbilidad entre ambos trastornos, hay pocos estudios empíricos que profundicen en las razones que pueden explicarla. Uno de los estudios más interesantes es el de Shafran (2002) en el que examina la relación que mantienen los TA con las creencias disfuncionales sobre las obsesiones las cuales, según los actuales modelos cognitivos, se hallan en la génesis y/o el mantenimiento del TOC (OCCWG, 1997, 2001, 2003, 2005). Los escasos estudios publicados sobre este aspecto coinciden en señalar una relación significativa entre la sintomatología de los TA y las creencias típicamente obsesivas, tanto en población general como en pacientes, si bien no se diferencia entre los diferentes subtipos o modalidades de TA (Humphreys et al., 2007; Freid, 2007; Lavender, Shubert, de Silva, y Treasure, 2006).

Este estudio se enmarca en la búsqueda de indicadores que permitan esclarecer la relación, así como las diferencias, que existen entre los TA y el TOC en aspectos psicopatológicos básicos. Específicamente, se busca examinar la influencia que tienen los síntomas TOC en la sintomatología TA a nivel cognitivo y metacognitivo. En consecuencia, los objetivos del presente trabajo fueron, primero, analizar en población general y pacientes con TA la relación entre los patrones o síntomas $\mathrm{O}-\mathrm{C}$ y los pensamientos intrusos sobre aspecto físico, ejercicio y dieta, así como sus consecuencias emocionales, valoraciones disfuncionales y estrategias para controlar dichos pensamientos. Y, segundo, examinar en población clínica si los síntomas y patrones O-C poseen alguna capacidad predictiva sobre la frecuencia con que las pacientes con TA experimentan intrusiones mentales sobre aspectos relacionados con estos trastornos, así como las valoraciones y estrategias de control asociadas a la intrusión que las propias pacientes consideran como más molesta (es decir, la que podría considerarse como más relevante desde el punto de vista clínico).

\section{MÉTODO}

\section{Participantes}

El estudio se ha realizado con dos grupos de participantes: uno de pacientes con diagnóstico principal de TA y otro de población general, con características socio-demográficas análogas. El grupo clínico estaba compuesto por 84 pacientes, todas ellas mujeres, con diagnóstico principal de TA (DSM-IV-TR; APA, 2000). $\mathrm{Su}$ edad media fue de 25,29 $(D T=8,87)$ años, y su índice de masa corporal (IMC) medio de $21,68(6,17)$. La mayoría eran solteras $(71,4 \%)$, tenían un nivel socio-económico medio $(63,1 \%)$, y estudios medios $(41,7 \%)$. En cuanto al subtipo de TA, 23 pacientes tenían diagnóstico de anorexia nerviosa restrictiva, 18 de anorexia nerviosa purgativa, 20 de bulimia nerviosa purgativa, 7 de bulimia nerviosa no purgativa, y 16 de trastorno alimentario no especificado. En el momento del estudio, 60 pacientes llevaban tratamiento ambulatorio, y las 24 restantes se encontraban en situación de ingreso hospitalario. Respecto a los diagnósticos secundarios, 20 de las 84 pacientes tenían un trastorno comórbido al TA en el momento del estudio, siendo la mayoría trastornos de personalidad (límite), del estado de ánimo (depresión mayor) y de ansiedad (trastorno de angustia con o sin agorafobia). Con el fin de evitar sesgos de interpretación de los datos, previamente se excluyeron del estudio 4 pacientes: 3 de ellas por presentar diagnóstico comórbido de TOC, y una con diagnóstico adicional de TOC de la personalidad. La muestra de población general se recabó una vez completada la de pacientes, con el objetivo de homogeneizar ambos grupos en variables socio-demográficas y en IMC. Este grupo estuvo formado por 100 mujeres, con una media de edad de 26,45 $(8,33)$ años y un IMC de 21,57 $(2,53)$. La mayoría eran solteras $(76 \%)$, con un nivel socio-económico medio $(63 \%)$ y estudios superiores $(51 \%)$.

\section{Instrumentos}

Inventario de Pensamientos Intrusos Alimentarios (INPIAS; Perpiñá et al., 2008). Se 
trata de un autoinforme formado por dos apartados. La primera parte evalúa a lo largo de 50 ítems la frecuencia con que la persona experimenta intrusiones sobre patrones de ingesta, ejercicio, e imagen corporal en una escala de 0 (nunca) a 6 (durante todo el día). Los 50 ítems se agrupan en 3 subescalas: Aspecto físico y dieta, Ejercicio físico, y Psicopatología alimentaria. En la segunda parte se evalúa la reacción emocional, la interferencia, y las valoraciones disfuncionales que ocasiona el pensamiento intruso alimentario considerado por la persona como más molesto y experimentado en los últimos tres meses (16 ítems) a través de las siguientes subescalas: Consecuencias emocionales y significado personal, y Fusión Pensamiento-Acción (FPA) y responsabilidad. Finalmente, se pide que indique las estrategias de afrontamiento que lleva a cabo con respecto a esa intrusión. Esta segunda parte consta de 17 ítems que se puntúan en una escala de 0 (nada/nunca) a 4 (extremadamente/siempre) y se agrupan en los siguientes factores: Estrategias del espectro de ansiedad, Supresión, Rituales TOC, Distracción, Hacer lo que dicta la intrusión, y No hacer nada. En un estudio anterior, la fiabilidad y validez del instrumento resultaron excelentes (Perpiñá, et al., 2008).

Inventario Obsesivo-Compulsivo de Clarky Beck (Clark-Beck Obsessive Compulsive Inventory, CBOCI; Clark y Beck, 2002). Instrumento de cribado para valorar la frecuencia de síntomas obsesivos y compulsivos. Está compuesto por 25 ítems que se responden en una escala tipo Likert de 4 puntos (0: «nunca»-3: "con mucha frecuencia»). Para este estudio se ha utilizado la adaptación y validación a población española de Belloch, Reina, García-Soriano, y Clark (2009).

Cuestionario de Actitudes hacia la Comida (The Eating Attitudes Test, EAT-26; Garner, Olmsted, Bohr y Garfinkel, 1982; versión abreviada del EAT-40 de Garner y Garfinkel, 1979). Evalúa actitudes y comportamientos relacionados con los TA, principalmente con la AN. Se obtiene una puntuación total, además de la de tres subescalas Hacer dieta Bulimia y preocupación por el alimento y Control oral. Se ha aplicado la adaptación española de Castro, Toro, Salamero y Guimerá (1991). Este cues- tionario fue completado únicamente por la muestra clínica.

The Restraint Scale (RS; Herman y Polivy, 1980) [Escala de Restricción]. Autoinforme que evalúa las fluctuaciones de peso, el grado de restricción y las actitudes hacia el peso y la comida. Consta de 10 items que se responden en una escala Likert de 4 ó 5 puntos según el item. El índice de fiabilidad obtenido en este estudio puede calificarse como bueno para la población no clínica $(\alpha=0,80)$, y aceptable para la de pacientes $(\alpha=0,65)$.

Inventario de Depresión de Beck (Beck Depression Inventory, BDI; Beck, Steer, y Brown, 1996). Versión española de Sanz, Perdigón, y Vázquez (2003). Este cuestionario evalúa a través de 21 ítems la intensidad o gravedad de los síntomas depresivos.

Inventario de ansiedad de Beck (Beck Anxiety Inventory, BAI; Beck y Steer, 1993. Versión española de Comeche et al., 1995). Autoinforme de 21 ítems que evalúa síntomas somáticos de ansiedad a través de una escala tipo Likert de 4 puntos.

\section{Procedimiento}

El grupo de población general estuvo compuesto por estudiantes de los últimos cursos de Psicología (Universidad de Valencia) y familiares y amigos de los mismos. No se incluyeron en el estudio personas que durante el año precedente hubiesen padecido algún trastorno mental que hubiera requerido atención especializada, o que no fueran capaces de comprender los cuestionarios, leer y/o escribir correctamente. Las pacientes pertenecían la Unidad de Trastornos Alimentarios del Hospital de San Juan (Alicante, España). Los diagnósticos fueron realizados por su psicólogo o psiquiatra siguiendo la Entrevista Clínica Estructurada del DSM-IV (First, Spitzer, Gibbon, y Williams, 1997).

Una vez se explicó a las participantes en qué consistía el estudio en general, se recabó su colaboración para participar en el mismo y dejaron constancia de ello firmando un consentimiento informado antes de completar el protocolo. Al finalizar, las participantes de la 
población general fueron pesadas y medidas por el entrevistador. El presente estudio recibió la aprobación ética por parte de los comités éticos del Hospital de San Juan y la Universidad de Valencia.

Con el objetivo de corroborar que no había diferencias entre la población general y el grupo de pacientes en variables socio-demográficas e IMC se llevaron a cabo pruebas de $t$ y de $\chi^{2}$ (muestras independientes) según la naturaleza de las variables. También se analizaron mediante pruebas $t$ (muestras independientes) las diferencias entre ambas muestras en síntomas obsesivos, depresión, ansiedad, y alimentarios, así como en los distintos factores del cuestionario sobre intrusiones mentales, INPIAS. Para analizar la relación entre las variables a estudiar se calcularon correlaciones de Pearson bivariadas, y parciales cuando había que controlar la influencia de terceras variables. Por otra parte, para estudiar si los síntomas TOC predecían las intrusiones alimentarias, sus valoraciones, consecuencias emocionales y estrategias de control asociadas, se llevaron a cabo varios análisis de regresión con el método de pasos sucesivos. Las variables dependientes fueron las subescalas del INPIAS. Como variables independientes se incluyeron la edad, el IMC, las puntuaciones de ansiedad y depresión y las subescalas del CBOCI y del EAT que previamente habían mostrado correlaciones significativas con el INPIAS.

\section{RESULTADOS}

Antes de comenzar con los análisis vinculados con los objetivos del estudio, se comprobó que no existían diferencias entre ambos grupos en las principales variables socio-demográficas analizadas: edad $\left(t_{(182)}=-0,91\right.$, n.s. $)$, estado civil $\left(\chi_{(3)}^{2}=0,72 ;\right.$ n.s. $)$, nivel socio-económico $\left(\chi_{(4)}^{2}\right.$ $=4,16$; n.s. $)$, y nivel de estudios $\left(\chi_{(2)}^{2}=5,34\right.$; n.s.). Además, ambas muestras fueron equiparables en cuanto a su IMC $\left(t_{(106,60)}=0,15 ;\right.$ n.s. $)$. Este dato no resulta sorprendente dado que el grupo de pacientes no era homogéneo en cuanto al subtipo de TA: como se comentó al describir la muestra, algo más de la mitad (43) tenían diagnóstico de Bulimia o TA no especificado, las cuales no presentaban bajo peso.
Por otra parte, la muestra de pacientes obtuvo como era de esperar puntuaciones significativamente más elevadas $(p<0,001)$ en las escalas de síntomas obsesivos (CBOCI: $t_{(126,}$, $\left.{ }_{44)}=8,51\right)$, depresivos $\left(\mathrm{BDI}: t_{(136,79)}=7,33\right)$, de ansiedad (BAI: $t_{(151,72)}=5,43$ ), y alimentarios (RS: $\left.t_{(165)}=9,09\right)$.

Respecto a las variables del cuestionario sobre intrusiones alimentarias (INPIAS), también el grupo clínico mostró mayores puntuaciones en todas las escalas $(p<0,001)$ : Aspecto físico y dieta $\left(t_{(182)}=9,20\right)$, Ejercicio físico $\left(t_{(182)}=3,93\right)$, Psicopatología alimentaria $\left(t_{(111,93)}=10,31\right)$, Consecuencias emocionales y significado personal $\left(t_{(175)}=12,99\right)$, FPA y responsabilidad $\left(t_{(94,59)}=10,20\right)$, Estrategias de ansiedad $\left(t_{(173)}=3,10\right)$, Supresión $\left(t_{(173)}=7,73\right)$, Rituales TOC $\left(t_{(125,69)}=5,19\right)$, Distracción $\left(t_{(173)}=1,72\right)$, Hacer lo que dicta la intrusión $\left(t_{(141,26)}=5,20\right)$ y No hacer nada $\left(t_{(173)}=2,24\right)$.

\section{Asociación entre síntomas obsesivo- compulsivos y pensamientos intrusos alimentarios}

El cálculo de las correlaciones bivariadas entre las escalas del CBOCI y las de INPIAS mostraron para ambas muestras un patrón similar, siendo significativas las asociaciones entre la mayoría de variables del INPIAS con las escalas de obsesiones y compulsiones, a excepción de la estrategia de control «No hacer nada» que no se asoció con las subescalas del CBOCI en ninguna de las dos muestras, y las estrategias típicas de ansiedad (evitación) y la de distracción en la muestra de pacientes (ver Tabla 1). En términos generales se observa que la presencia de obsesividad elevada (subescala de obsesiones del CBOCI) se asocia especialmente con la frecuencia con que se experimentan intrusiones relacionadas con la dieta, el ejercicio y el aspecto físico, y en mucha menor medida con las intrusiones más vinculadas a la patología alimentaria per se (i.e., impulsos de vomitar, ayunar, auto-instrucciones de no comer, etc.). Con todo, es interesante señalar que esas intrusiones más patológicas se asocian con puntuaciones elevadas tanto en obsesiones como en compulsiones en la muestra clínica, pero solo 
con obsesiones en la muestra de población general. Sin embargo, las consecuencias emocionales y valoraciones disfuncionales de la intrusión alimentaria más molesta sí que se asociaron, en ambas muestras, con puntuaciones elevadas en obsesiones y en compulsiones, es decir, con la presencia de psicopatología O-C. En cuanto a las estrategias de control/ neutralización ante la intrusión más molesta, los perfiles correlacionales son bastante distintos en ambas muestras: mientras que en las pacientes las asociaciones más evidentes con sintomatología O-C se produjeron con el uso de rituales característicos del TOC y con el uso de la supresión ante una intrusión alimentaria molesta, en la población no clínica las asociaciones entre síntomas O-C y utilización de estrategias de control ante intrusiones alimentarias molestas fueron más numerosas e inespecíficas (ver Tabla 1).
A continuación realizamos correlaciones parciales en las que se introdujeron como controles la edad, el BDI y el BAI, dadas las diferencias previamente encontradas entre los grupos. Se introdujo también el IMC pues, aunque no se detectaron diferencias entre los grupos, el bajo peso ha sido señalado como una posible causa de la obsesividad presente en las pacientes con TA (Garfinkel y Garner, 1982; Serpell, et al., 2002).

Los resultados de estas correlaciones parciales revelaron la anulación de un buen número de las asociaciones previamente encontradas en ambas muestras. En el caso de la población general solo se mantuvieron las relaciones entre obsesividad elevada (CBOCI) y las intrusiones sobre ejercicio físico y dieta, mientras que en el grupo de pacientes no hubo ninguna relación significativa. Sin embargo, las asociaciones entre la valoración disfuncional de Fusión pensamiento-acción de la intrusión más molesta y la

Tabla 1. Correlaciones bivariadas y parciales (controlando edad, IMC, depresión y ansiedad) entre CBOCI e INPIAS en población general y pacientes por separado

\begin{tabular}{|c|c|c|c|c|c|c|c|c|}
\hline \multirow{5}{*}{ INPIAS } & \multicolumn{4}{|c|}{ Población general $(n=100)$} & \multicolumn{4}{|c|}{ Pacientes $(n=84)$} \\
\hline & \multicolumn{8}{|c|}{ Inventario Clark-Beck de Obsesiones-Compulsiones (CBOCI) } \\
\hline & Obs. & Comp. & Obs. & Comp. & Obs. & Comp. & Obs. & Comp. \\
\hline & \multicolumn{4}{|c|}{$\begin{array}{c}\text { Control edad, IMC, } \\
\text { BDI, BAI }\end{array}$} & & & \multicolumn{2}{|c|}{$\begin{array}{c}\text { Control edad, IMC, } \\
\text { BDI, BAI }\end{array}$} \\
\hline & \multicolumn{6}{|c|}{ Frecuencia de pensamientos intrusos } & & \\
\hline Aspecto y dieta & $0,33 * *$ & $0,30 * *$ & 0,08 & 0,16 & $0,48 * *$ & $0,37 * *$ & 0,24 & 0,15 \\
\hline Ejercicio físico & $0,36 * *$ & $0,35 * *$ & $0,23 *$ & $0,27 * *$ & $0,40 * *$ & $0,38 * *$ & 0,18 & 0,16 \\
\hline PA & $0,24 *$ & 0,15 & $-0,05$ & $-0,03$ & $0,27 *$ & $0,22 *$ & 0,19 & 0,17 \\
\hline \multicolumn{9}{|c|}{ Consecuencias emocionales y valoraciones disfuncionales } \\
\hline Consecuencias & $0,40 * *$ & $0,34 * *$ & 0,18 & $0,21 *$ & $0,44 * *$ & $0,27 *$ & $0,31 *$ & 0,14 \\
\hline FPA-R & $0,32 * *$ & $0,28 * *$ & $0,25^{*}$ & $0,23 *$ & $0,61 * *$ & $0,51 * *$ & $0,44 * *$ & $0,31 *$ \\
\hline \multicolumn{9}{|c|}{ Estrategias de control } \\
\hline Ansiedad & $0,32 * *$ & $0,32 * *$ & 0,18 & $0,23 *$ & 0,17 & 0,13 & 0,19 & 0,11 \\
\hline Supresión & $0,36 * *$ & $0,34 * *$ & $0,21 *$ & $0,24 *$ & $0,28 *$ & $0,24 *$ & 0,15 & 0,09 \\
\hline Rituales TOC & 0,12 & $0,33 * *$ & 0,09 & $0,32 * *$ & $0,27 *$ & $0,41 * *$ & 0,16 & $0,32 * *$ \\
\hline Distracción & $0,32 * *$ & 0,19 & 0,18 & 0,17 & 0,03 & 0,03 & 0,05 & 0,04 \\
\hline Hacer dieta & $0,29 * *$ & $0,27 * *$ & 0,18 & 0,20 & 0,17 & $0,29 *$ & 0,05 & 0,23 \\
\hline No hacer nada & 0,12 & 0,10 & 0,08 & 0,07 & $-0,07$ & $-0,02$ & $-0,14$ & $-0,07$ \\
\hline
\end{tabular}

Obs.: Obsesiones; Comp.: Compulsiones. IMC: Índice de Masa Corporal; BDI: Inventario de Depresión de Beck; BAI: Inventario de Ansiedad de Beck; PA: Psicopatología alimentaria; FPA-R: Fusión Pensamiento-Acción y Responsabilidad. *p $<0,05 ;{ }^{* *} \mathrm{p}<0,01$; 
presencia de síntomas obsesivos y compulsivos (CBOCI) siguió siendo importante en ambas muestras. Por su parte, las consecuencias emocionales generadas por dicha intrusión, se mantuvieron asociadas con síntomas compulsivos en la muestra no clínica, y con síntomas obsesivos en las pacientes con TA. Respecto a las estrategias de control, la puntuación en síntomas compulsivos mantuvo sus asociaciones con las estrategias de ansiedad, la supresión, y los rituales TOC en la muestra de población general, mientras que en la de pacientes únicamente siguió siendo significativa con la supresión (Tabla 1).

\section{Variables que predicen las intrusiones}

alimentarias, sus consecuencias emocionales, valoraciones disfuncionales y estrategias de control, en pacientes alimentarias.

Tras comprobar que existía una asociación significativa entre las variables del estudio, y con el fin de analizar el papel de la sintomatología obsesiva en la psicopatología alimentaria, se procedió a examinar qué variables podían predecir la experimentación de intrusiones alimentarias, así como el tipo de valoraciones y estrategias de control que suscitan, en la muestra de pacientes.

Los análisis de regresión mostraron que la frecuencia de intrusiones alimentarias sobre aspecto físico y dieta se explicaban en su mayor parte por el factor Dieta del EAT (58\%), y en una proporción mucho menor, por la sintomatología depresiva (BDI) y el factor Bulimia del EAT (6\% y $3 \%$ de la varianza total explicada, respectivamente). A su vez, la frecuencia de intrusiones sobre ejercicio físico fueron explicadas también por el factor Dieta $(51 \%$ de la varianza total), y la frecuencia de intrusiones más patológicas se explicaban básicamente por el factor Bulimia (52\%). Sin embargo, las consecuencias emocionales provocadas por la intrusión escogida por las pacientes como más

Tabla 2. Análisis de regresión en pacientes alimentarias. VD: escalas del INPIAS; VI: Edad, IMC, depresión, ansiedad, EAT, y escalas del CBOCI.

\begin{tabular}{|c|c|c|c|c|c|c|}
\hline VD & VI & Cambio $R^{2}$ & $F$ & $g l$ & $\beta$ & $t$ \\
\hline \multicolumn{7}{|c|}{ INPIAS-Parte 1. Frecuencia de pensamientos intrusos } \\
\hline \multirow[t]{3}{*}{ Aspecto/Dieta } & EAT. Dieta & 0,58 & $97,20 * * *$ & 1,70 & 0,40 & $4,66 * * *$ \\
\hline & BDI & 0,06 & $62,41 * * *$ & 2,69 & 0,29 & $3,95 * * *$ \\
\hline & EAT.Bulimia & 0,03 & $47,20 * * *$ & 3,68 & 0,25 & $2,57 *$ \\
\hline Ejercicio físico & EAT.Dieta & 0,51 & $74,63 * * *$ & 1,70 & 0,72 & $8,64 * * *$ \\
\hline PA & EAT.Bulimia & 0,52 & $76,14 * * *$ & 1,70 & 0,72 & $8,72 * * *$ \\
\hline \multicolumn{7}{|c|}{ INPIAS-Parte 2-A .Consecuencias emocionales y valoraciones disfuncionales } \\
\hline \multirow[t]{2}{*}{ Consecuencias } & EAT. Bulimia & 0,22 & $19,30 * * *$ & 1,67 & 0,34 & $2,92 * * *$ \\
\hline & CBOCI. Obs. & 0,07 & $13,56 * * *$ & 2,66 & 0,29 & $2,51^{*}$ \\
\hline \multirow[t]{2}{*}{ FPA-R } & CBOCI. Obs. & 0,35 & $36,61 * * *$ & 1,67 & 0,52 & $5,32 * * *$ \\
\hline & Edad & 0,06 & $23,68 * * *$ & 2,66 & $-0,26$ & $-2,70 * *$ \\
\hline \multicolumn{7}{|c|}{ INPIAS-Parte 2-B. Estrategias de control } \\
\hline Supresión & Edad & 0,07 & $6,49 * *$ & 1,65 & $-0,30$ & $-2,55^{*}$ \\
\hline \multirow[t]{2}{*}{ Rituales TOC } & CBOCI-Com. & 0,18 & $13,84 * * *$ & 1,65 & 3,63 & $3,24 * *$ \\
\hline & IMC & 0,06 & $10,01 * * *$ & 2,64 & $-0,26$ & $-2,30 *$ \\
\hline
\end{tabular}

AT: Eating Attitudes Test. BDI: Inventario de Depresión de Beck; CBOCI: Inventario Clark-Beck de Obsesiones-Compulsiones; Obs.: Obsesiones. Comp.: Compulsiones. IMC: Índice de Masa Corporal; PA: Psicopatología alimentaria; FPA-R: Fusión Pensamiento-Acción y Responsabilidad; VD: variables dependientes; VI: variables independientes.

$* p<0,05 ; * p<0,01 ; * p<0,001$. 
molesta, fueron explicadas no solo por la presencia de psicopatología alimentaria (Bulimia: $22 \%$ ), sino también por sintomatología obsesiva (CBOCI: 7\%). La importancia de la psicopatología obsesiva (CBOCI-Obsesiones) se mostró aun más notable a la hora de predecir la presencia de la valoración disfuncional de fusión pensamiento-acción ante la intrusión alimentaria más desagradable $(35 \%$ de varianza explicada). Finalmente, por lo que se refiere a las estrategias empleadas para controlar la intrusión más molesta, la utilización de estrategias de Supresión fue explicado sobre todo por la edad (7\%), mientras que el uso de Rituales TOC se explicó especialmente por la presencia de sintomatología compulsiva (CBOCI-Compulsiones: $18 \%$ ). Las estrategias de control de tipo ansioso y la distracción no se introdujeron en los análisis, dado que los análisis correlacionales previos no mostraron asociación alguna con las medidas del estudio en el grupo de pacientes. Los resultados se encuentran en la Tabla 2.

\section{DISCUSIÓN}

Este estudio analiza la asociación entre la sintomatología TOC y variables cognitivas relacionadas con la psicopatología alimentaria. El primer objetivo fue examinar en población general y pacientes con TA la relación entre sintomatología O-C y las intrusiones mentales de contenido alimentario, así como sus consecuencias emocionales, valoraciones disfuncionales, y estrategias para controlarlas. Los resultados sugieren que cuanto más importante es la sintomatología obsesivo-compulsiva, más frecuentemente se experimentan intrusiones alimentarias, tanto en población general como en personas con un diagnóstico de TA. Esta relación es análoga a la que se produce cuando lo que se examina es la asociación entre síntomas $\mathrm{O}-\mathrm{C}$ y la frecuencia de intrusiones con contenido análogo a las obsesiones (p.ej., Morillo, Be1loch, y García-Soriano, 2007; Morillo et al., 2003; Purdon, y Clark, 1993; 1994).

No obstante, una diferencia sustancial entre los resultados de los estudios con intrusiones obsesivas y los encontrados en el presente con las intrusiones alimentarias, radica en que en el primer caso los resultados se mantienen estables cuando se controlan los niveles de ansiedad y depresión. Sin embargo, los resultados de este estudio indican que una buena parte de las relaciones previamente observadas está mediatizada por otras variables: la edad, el IMC, la ansiedad, y la depresión. Es decir, que cuando se experimentan intrusiones con contenido alimentario, la presencia concomitante de sintomatología obsesiva se debe más bien al efecto de otras variables no estrictamente relacionadas con la psicopatología alimentaria. De hecho, la edad y el IMC han mostrado tener un papel relevante en los TA (p.ej., Benedito, Perpiñá, Botella, y Baños, 2003; Grilo, 2006; Fairburn, 2008). En cuanto a los síntomas depresivos y ansiosos, hay bastantes estudios que evidencian asociaciones entre la patología alimentaria y el afecto negativo (Stice, 2001; Whiteside et al., 2007). En conjunto, por tanto, nuestros datos sugieren que la presencia de síntomas TOC en personas con TA se debe primariamente a variables comunes a ambos conjuntos de trastornos (especialmente, ansiedad y depresión), y no tanto a una comorbilidad de base entre el TOC $\mathrm{y} \operatorname{los} \mathrm{TA}$.

Cuando examinamos la relación entre síntomas de TOC con la repercusión emocional que tiene la intrusión alimentaria más molesta y cómo se valora, los resultados son muy diferentes a cuando se tiene en cuenta únicamente la frecuencia de intrusiones: tanto si se controlan los potenciales efectos mediadores de la edad, el IMC, la ansiedad y la depresión, como si no se controlan, los síntomas TOC se asocian con una mayor tendencia a valorar una intrusión alimentaria de manera disfuncional. Y esta tendencia se produce tanto en personas sin patología alimentaria ni obsesiva, como en pacientes con un diagnóstico formal de TA. La importancia de la valoración disfuncional de FPA, que es la más representada en el INPIAS, es congruente con el planteamiento de Shafran (2002) en torno a la relevancia de las valoraciones disfuncionales típicamente obsesivas para los TA, y permite comprender mejor el tipo de relacines que puede haber entre estos trastornos y el TOC. También las consecuencias emocionales negativas provocadas por una intrusión alimentaria molesta se mantienen cuando se 
controla el peso de las variables antes mencionadas, pero en esta ocasión las asociaciones con los síntomas O-C son diferentes según la población analizada: en el caso de las personas sin patología mental, la repercusión emocional negativa de la intrusión molesta se asocia a una mayor tasa de síntomas compulsivos, mientras que en las personas con TA se relaciona con un incremento de síntomas obsesivos.

En cuanto al papel que juegan las estrategias de control/neutralización que suscita la intrusión alimentaria más molesta sobre la sintomatología O-C, las más relevantes son, sin duda, las que en el INPIAS se agrupan bajo la etiqueta de «rituales TOC», es decir, estrategias como ordenar y comprobar. La asociación de estas estrategias con la puntuación en sintomatología compulsiva se mantuvo en ambas muestras independientemente de que se controlara o no el peso de posibles variables mediadoras. Este resultado coincide con el hecho de que la sintomatología obsesivo-compulsiva que se observa en las pacientes TA con más frecuencia es de subtipo orden y simetría (Bastiani et al., 1996; Matsunaga et al., 1999a; Matsunaga et al., 1999b; Halmi et al., 2003). Sin embargo, a diferencia de los resultados obtenidos por Radomsky, de Silva, Treasure, y Murphy (2002), en los que el empleo de la estrategia de neutralización en pacientes con TA se asoció significativamente con sintomatología TOC, en este estudio el factor Supresión (que incluye la estrategia de neutralización) sólo estuvo asociado a los síntomas TOC en el caso de la población general, pero no en la muestra de pacientes con TA. Este resultado diferente podría deberse al hecho de que el factor «Supresión» incluye, tal y como lo evalúa el INPIAS, estrategias disfuncionales adicionales como la evitación, enfadarse consigo mismo, o formar en la mente otro pensamiento o imagen que contrarreste el malestar provocado por el pensamiento, por lo que la asociación entre la supresión propiamente dicha y la obsesividad puede quedar diluida.

Un resultado interesante que merece un comentario específico es el obtenido con la estrategia «no hacer nada» que no mostró relación significativa con la puntuación en obsesiones ni con la de compulsiones. Esta ausencia de asociación es coherente con uno de los supuestos en los que se asienta, por ejemplo, el tratamiento cognitivo del TOC en el que, entre otras cosas, se anima al paciente a no luchar activamente contra sus obsesiones, a no hacer nada para controlarlas, razón por la cual se puede considerar como una estrategia adaptativa (Kavanagh, Andrade, y May, 2005). De hecho, la correlación entre «no hacer nada» y síntomas TOC en las pacientes fue negativa (aunque solo marginalmente significativa), lo que indica que a más sintomatología TOC mayor es la necesidad de hacer algo con los pensamientos para controlarlos.

El segundo objetivo del estudio era analizar en qué medida los síntomas O-C podían predecir la frecuencia de intrusiones alimentarias, sus valoraciones disfuncionales, y las estrategias de control o neutralización empleadas. Para estudiarlo tuvimos en cuenta únicamente a la muestra de pacientes con TA. Tomados en su conjunto, los resultados indican, primero, que la frecuencia de intrusiones alimentarias depende, fundamentalmente, de la sintomatología propia de los TA, tal y como se evalúa con el EAT; segundo, que la presencia de obsesividad (subescala de obsesiones del CBOCI) predice el hecho de que una intrusión alimentaria molesta tenga un impacto emocional negativo en una paciente con TA y sea valorada de un modo claramente disfuncional; y, tercero, que la presencia de compulsiones (subescala del CBOCI) explica, junto con el valor del IMC, que las pacientes pongan en marcha estrategias activas de control típicamente obsesivo-compulsivas para neutralizar el impacto de una intrusión molesta de temática alimentaria.

Estos datos son coherentes con los observados en los análisis correlaciones previos. La frecuencia con la que las pacientes tienen intrusiones con contenido alimentario no se puede predecir a partir de sus niveles de obsesividad, sino, precisamente, a partir de su sintomatología alimentaria. Es decir, que en pacientes alimentarias la presencia de síntomas TOC no tiene un papel significativo en la experimentación de pensamientos intrusos con contenido específicamente relacionado con TA. Por tanto, parece que son las consecuencias de tener una intrusión molesta de contenido alimentario, y no la frecuencia con la que se tienen intrusiones con 
estos contenidos, lo que parece establecer un puente entre el TOC y los TA. Esta idea es coherente con la descripción de Rachman y Shafran (1999) sobre la importancia de las creencias disfuncionales de Fusión Pensamiento-Acción en pacientes con TA, creencias que a su vez se han asociado a la sintomatología obsesiva en estas pacientes (Radomsky et al., 2002). Finalmente, resulta destacable que la sintomatología compulsiva sea predicha por el IMC, además de por la presencia de estrategias de control ritualizadas características del TOC (comprobar, ordenar o limpiar). La aparición del IMC en esta ecuación es coherente con estudios en los que se ha encontrado una asociación entre el bajo peso y la sintomatología O-C (Milos et al., 2002). Un resultado inesperado fue que las estrategias de «Supresión» fueron explicadas por la edad (7\%) con una asociación negativa. Esto podría explicarse por el hecho de que las pacientes más jóvenes traten (todavía) de luchar contra sus intrusiones mentales tratando de suprimirlas o neutralizarlas, estrategias típicamente empleadas en los pacientes con TOC, mientras que las pacientes de más edad, y por tanto, con un TA probablemente crónico, se «dejan llevar por sus pensamientos». Estos datos deberán ser analizados más específicamente en posteriores estudios. Por ejemplo, sería interesante analizar qué variables predicen las estrategias para controlar los pensamientos intrusos alimentarios. Siguiendo los modelos cognitivos sobre el procesamiento de las intrusiones mentales de tipo obsesivo (p.ej., Clark, 2004: Rachman, 1997, 1998; Salkovskis, 1999) y la teoría sobre la elaboración de las intrusiones (Kavanagh et al., 2005; May, Andrade, y Panabokke, 2004), podría hipotetizarse que las propias valoraciones disfuncionales y las consecuencias emocionales de las intrusiones son las responsables de un empleo más frecuente de estrategias de control, y específicamente de las estrategias más disfunciones como la supresión.

En conclusión y según nuestros resultados, los síntomas obsesivos no influyen de forma determinante en el contenido ni en la frecuencia con la que se experimentan intrusiones mentales desagradables de contenido alimentario, sino más bien en cómo se valoran esas intrusiones, las consecuencias emocionales que tienen para la persona, y el tipo de estrategias de control/neutralización que suscitan. En cambio, son los síntomas alimentarios los que tienen un mayor peso en el contenido y la frecuencia de las intrusiones, como cabía esperar.

Una de las limitaciones del estudio viene dada por el empleo de auto-informes para la recogida de los datos, puesto que no podemos asegurar la ausencia de sesgos (Gallego et al., 2007). Además, hubiera sido interesante poder contar con una muestra clínica más amplia que hubiera permitido realizar un estudio diferencial a nivel de subtipos de TA a fin de establecer un patrón más específico en la relación entre los TA y el TOC. Tampoco se ha tenido en cuenta la gravedad del TA ni su cronicidad, variables que presumiblemente pueden explicar algunos de los resultados encontrados. Desde esta perspectiva, el hecho de que el grupo de pacientes con TA no difiera de los controles normales en el IMC, puede resultar una limitación adicional. No obstante, como ya se comentó al indicar este dato, la ausencia de diferencias se debe a que el diagnóstico de algo más de la mitad de las pacientes conllevaba ausencia de bajo peso. En nuestra opinión este hecho puede ser considerado más que como una limitación como un elemento que favorece la posibilidad de comparación de personas con TA y personas sin el trastorno. En todo caso, se ha intentado controlar introduciendo el IMC como co-variable en los análisis realizados.

En suma, este estudio pretende contribuir al conocimiento de la relación entre los TA y el TOC. Estos primeros resultados ponen de manifiesto que el contenido ideativo de las intrusiones y de los fenómenos cognitivos que se dan en los TA son específicos de estos trastornos. Pero a la vez indican que hay un transfondo de «obsesividad» en la forma en que tales contenidos se valoran y se tratan de controlar.

\section{REFERENCIAS}

American Psychiatric Association (2000). Diagnostic and Statistical Manuel of Mental Disorders, 4th Ed, Text Revision (DSM-IV-TR). Washington, D.C., American Psychiatric Association. 
Bastiani, A. M., Altemus, M., Pigott, T. A., Rubenstein, C., Weltzin, T. E., y Kaye, W. H. (1996). Comparison of obsessions and compulsions in patients with Anorexia Nervosa and Obsessive Compulsive Disorder. Biological Psychiatry, 39, 966-969.

Beck, A. T., y Steer, R. A. (1993). Manual for the Beck Anxiety Inventory. San Antonio, TX: Psychological Corporation.

Beck, A.T., Steer, R.A., y Brown, G.K. (1996). Manual of the Beck Depression Inventory-Second Edition. San Antonio, TX: The Psychological Corporation.

Belloch, A., Reina, N., García-Soriano, G., y Clark, D.A. (2009). El Inventario Clark-Beck de Obsesión-Compulsión (C-BOCI): Validación para su uso en población española. Revista de Psicopatología y Psicología Clínica, 14, 95-105.

Benedito, M.C., Perpiná, C., Botella, C., y Baños, R. (2003). Imagen corporal y restricción alimentaria en adolescentes. Anales de Pediatría, 58, 268-272.

Carter, J.C., Blackmore, E., Sutandar-Pinnock, K., y Woodside, D.B. (2004). Relapse in anorexia nervosa: a survival analysis. Psychological Medicine, 34, 671-679.

Castro, J., Toro, J., Salamero, M., y Guimerá, E. (1991). The Eating Attitudes Test: Validation of the Spanish version. Psychological Assessment, 7, 175-179.

Clark, D. A. (2004). Cognitive-behavioural therapy for $O C D$. New York: The Guilford Press.

Clark, D. A., y Beck, A. T. (2002). Manual for the ClarkBeck Obsessive Compulsive Inventory. San Antonio, TX: Psychological Corporation.

Comeche, M.I., Díaz, M.I., y Vallejo, M.A. (1995). Cuestionarios, Inventarios y Escalas. Ansiedad, Depresión y Habilidades Sociales. Madrid: UNED, Fundación Universidad-Empresa.

Crane, A.M., Roberts, M.E., y Treasure, J. (2007). Are obsessive-compulsive personality traits associated with a poor outcome in anorexia nervosa? A systematic review of randomized controlled trials and naturalistic outcome studies. International Journal of Eating Disorders, 40, 581-588.

Davies, H., Liao, T., Campbell, I., y Tchanturia, K. (2009) Multidimensional self reports as a measure of characteristics in people with eating disorders. Weight and Eating Disorders, 14, 84-91.

Fairburn, C. (2008). Cognitive behavior therapy and eating disorders. New York: The Guildford Press.

First, M.B., Spitzer, R.L., Gibbon, M.G., y Williams, J.B. (1997). Structured clinical interview for DSM-IV axis I disorders (SCID-I), Clinician version. Washington, DC: American Psychological Association.

Freid, C.M. (2007). Beliefs associated with eating disorders and obsessive compulsive disorder: The development of the Obsessive Beliefs about Body Size and Eating Survey (OBBSES). Dissertation. University of Vanderbilt.
Gallego, M.J., Botella, C., Quero, S., Baños, R.M., y García-Palacios, A. (2007). Propiedades psicométricas de la Escala de Miedo a la Evaluación Negativa (BFNE) en nuestra clínica. Revista de Psicopatología y Psicología Clínica, 12, 136-176.

García-Soriano, G, Belloch, A., y Morillo, C. (2008). Sobre la heterogeneidad del trastorno obsesivo-compulsivo: una revisión. Revista de Psicopatología y Psicología Clínica, 13, 65-84.

Garfinkel, P.E., y Garner, D.M. (1982). Anorexia Nervosa: A Multidimensional Perspective. Brunner Mazel: New York.

Garner D.M., y Garfinkel P.E. (1979). The Eating Attitudes Test: an index of the symptoms of anorexia nervosa. Psychological Medicine, 9, 273-279.

Garner, D.M., Olmsted, M.P., Bohr, Y., y Garfinkel, P.E. (1982). The eating attitudes test: Psychometric features and clinical correlates. Psychological Medicine, 12, 871-878.

Grilo, C.M. (2006). Eating and weight disorders. Clinical Psychology: A modular course. New York: Psychology Press.

Halmi, K.A., Sunday, S.R., Klump, K.L., Strober, M., Leckman, J.F., Fichter, M., Kaplan, A., Woodside, B., Treasure, J., Berrettini, W.H., Shabboat, M.A., Bulik, C.M., y Kaye, W.H. (2003). Obsessions and compulsions in anorexia nervosa subtypes. International Journal of Eating Disorders, 33, 308-319.

Herman, C.P., y Polivy, J. (1980). Restrained eating. En Stunkard, A.J. (Ed.), Obesity. Filadelfia, P.A. Saunders.

Humphreys, J.D., Clopton, J.R. y Reich, D.A. (2007). Disordered eating behaviour and obsessive compulsive symptoms in college students: Cognitive and affective similarities. Eating Disorders: The Journal of Treatment and Prevention, 15, 247-259.

Kavanagh, D.J., Andrade, J., y May, J. (2005). Imaginary relish and exquisite torture: The elaborated intrusion theory of desire. Psychological Review, 112, 446-467.

Lavender, A., Shubert, I., de Silva, P., y Treassure, J. (2006). Obsessive-compulsive beliefs and magical ideation in eating disorders. British Journal of Clinical Psychology, 45, 331-342.

Matsunaga, H., Kiriike, N., Iwasaki, Y., Miyata, A., Yagamati, S., y Kaye, W.H. (1999a). Clinical characteristics in patients with anorexia nervosa and obsessive-compulsive disorder. Psychological Medicine, 29, 407-414.

Matsunaga, H., Miyata, A., Iwasaki, Y., Matsui, T., Fujimoto, K., y Kiriike, N. (1999b). A comparison of clinical features among Japanese eating-disordered women with obsessive compulsive disorder. Comprehensive Psychiatry, 40, 337-342.

May, J., Andrade, J. y Panabokke, N. (2004). Images of desire: Cognitive models of craving. Memory, 12, 447461. 
Milos, G., Spindler, A., Ruggiero, G., Klaghofer, R., y Schnyder, U. (2002). Comorbidity of obsessive-compulsive disorders and duration of eating disorders. International Journal of Eating Disorders, 31, 284-9.

Morgan, J., Wolf, B., Metzger, E.D., y Jimerson, D.C. (2007). Obsessive-compulsive characteristics in women who have recovered from bulimia nervosa. International Journal of Eating Disorders, 40, 381-385.

Morillo, C., Belloch, A., y García-Soriano, G. (2007). Clinical Obsessions in obsessive-compulsive patients and obsession-relevant intrusive thoughts, in non-clinical, depressed and anxious subjects: Where are the differences? Behavioural Research and Therapy, 45, 1319-1333.

Morillo, C., Giménez, A., Belloch, A., Lucero, M., Carrió, C. y Cabedo, E. (2003). Pensamientos intrusos en obsesivos subclínicos: Contenidos, valoraciones, y estrategias de control. Revista de Psicopatología y Psicología Clínica, 8, 23-28.

Obsessive-Compulsive Cognitions Working Group. (1997). Cognitive assessment of obsessive-compulsive disorder. Behaviour Research and Therapy, 35, 667681.

Obsessive-Compulsive Cognitions Working Group. (2001). Development and initial validation of the obsessive beliefs questionnaire and the interpretation of intrusions inventory. Behaviour Research and Therapy, 39, 987-1006.

Obsessive-Compulsive Cognitions Working Group. (2003). Psychometric validation of the Obsessive Beliefs Questionnaire and the Interpretation of Intrusions Inventory: Part I. Behaviour Research and Therapy, 41, 863-878.

Obsessive-Compulsive Cognitions Working Group. (2005). Psychometric validation of the Obsessive Beliefs Questionnaire and the Interpretation of Intrusions Inventory-Part II: Factor analyses and testing of a brief version. Behaviour Research and Therapy, 43, 15271542.

Perpiñá, C., Roncero, M., y Belloch, A. (2008). Eatingrelated intrusions in a community sample: Development and validation of the eating-related intrusive thoughts INPIAS. Revista de Psicopatología y Psicología Clínica, 13, 137-158.

Pollice, C., Kaye, W.H., Greeno, C., y Weltzin, T.E. (1997). Relationship of depression, anxiety, and obsessionality to state of illness in anorexia nervosa. International Journal of Eating Disorders, 21, 367-376.

Purdon, C., y Clark, D. (1993). Obsessive intrusive thoughts in non-clinical subjects. Part I. Content and relation with depressive, anxious, and obsessional symptoms. Behaviour Research and Therapy, 31, 713720.

Purdon, C., y Clark, D. (1994). Obsessive Intrusive thoughts in nonclinical subjects. Part II. Cognitive appraisal, emotional response and thought control strategies. Behaviour Research and Therapy, 32, 403-410.

Rachman, S. (1997). A cognitive theory of obsessions. Behaviour Research and Therapy, 35, 793-802.

Rachman, S. (1998). A cognitive theory of obsessions: Elaborations. Behaviour Research and Therapy, 36, 385-401.

Rachman, S., y Shafran, R. (1999). Cognitive distortions: Thought-action fusion. Clinical Psychology and Psychotherapy, 6, 80-85.

Rachman, S., y de Silva, P. (1978). Abnormal and normal obsessions. Behaviour Research and Therapy, 16, 233248.

Radomsky, A.S., de Silva, P., Todd, G., Treasure, J., y Murphy, T. (2002). Thought-shape fusion in anorexia nervosa: an experimental investigation. Behaviour Research and Therapy, 40, 1169-1177.

Salkovskis, P.M. (1999). Understanding and treating obsessive-compulsive disorder. Behaviour Research and Therapy, 37, 29-52.

Sánchez-Reales, S. (2009). Los trastornos de la conducta alimentaria en el espectro obsesivo compulsivo: Análisis de variables cognitivas y de personalidad. Tesis doctoral no publicada. Universidad de Valencia.

Sanz, J., Perdigón, A.L., y Vázquez, C. (2003). Adaptación Española del Inventario para la Depresión de Beck II (BDI-II): 2. Propiedades psicométricas en población general. Clínica y Salud, 14, 249-280.

Serpell, L., Livingstone, A., Neiderman, M., y Lask, B. (2002). Anorexia nervosa: Obsessive-compulsive disorder, obsessive-compulsive personality disorder, or neither? Clinical Psychology Review, 22, 674-669.

Serpell, L., Treasure, J., Teasdale, J., y Sullivan, V. (1999). Anorexia nervosa: Friend or Foe? International Journal of Eating Disorders, 25, 177-186.

Shafran, R. (2002). Eating disorders and obsessive compulsive disorder. En Frost, R. y Steketee, S. (Eds.). Cognitive approaches to obsessions and compulsions: Theory, assessment, and treatment. (pg. 224). Oxford: Elsevier.

Soetens, B., Braet, C., Dejonckheere, P., y Roets, A. (2006). «When suppression backfires». The ironic effects of suppressing eating-related thoughts. Journal of Health Psychology, 11, 655-668.

Speranza, M., Corcos, M., Godart, N., Loas, G., Guilbaud, O., Jeammet, P., y Flament, M. (2001). Obsessive compulsive disorders in eating disorders. Eating Behaviors, 2, 193-207.

Stice, E. (2001). A prospective test of the dual pathway model of bulimic pathology: Mediating effects of dieting and negative affect. Journal of Abnormal Psychology, 110, 124-135.

Swinbourne, J.M., y Touyz, S.W. (2007). The co-morbidity of eating disorders and anxiety disorders: A review. European Eating Disorders Review, 15, 253-274. 
Thiel, A., Zuger, M., Jacoby, G.E., y Schubler, G. (1998). Thirty-month outcome in patients with anorexia nervosa or bulimia nervosa and concominant obsessivecompulsive disorder. American Journal of Psychiatry, 155, 244-249.

Thornton, C., y Russell, J. (1995). Obsessive compulsive comorbidity in the dieting disorders. International Journal of Eating Disorders, 21, 83-87.

Von Ranson, K.M., Kaye, W.H., Weltzin, T.E., Rao, R., y Matsunaga, H. (1999). Obsessive-compulsive disorder symptoms before and after recovery from bulimia nervosa. American Journal of Psychiatry, 156, 1703-1708.
Wegner, D.M., Schneider, D.J., Carter, S.R., y White, T.L. (1987). Paradoxical effects of thought suppression. Journal of Personality and Social Psychology, 53, 5-13.

Whiteside, U., Chen, E., Neighbors, C., Hunter, D., Lo, T., y Larimer, M. (2007). Difficulties regulating emotions: Do binge eaters have fewer strategies to modulate negative affect? Eating Behaviours, 8, 162-169.

Woolrich, R.A., Cooper, M.J., y Turner, H.M. (2008). Metacognition in patients with anorexia nervosa, dieting and non-dieting women: A preliminary study. European Eating Disorders Review, 16, 11-20. 